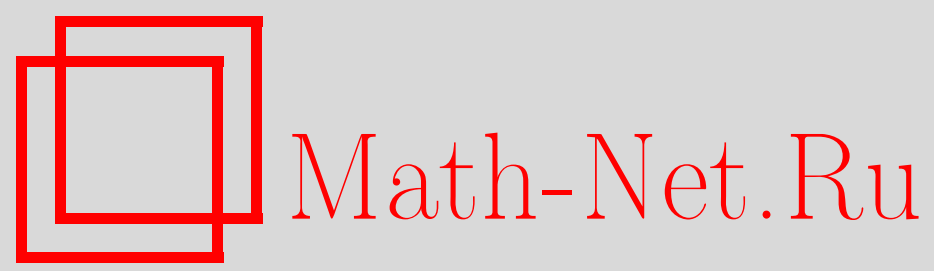

О. И. Сидорова, Об одном применении дробного движения Леви к моделированию сетевого трафика, Вестник ТвГУ. Серия: Прикладная математика, 2017, выпуск 1, 17-29

DOI: https://doi.org/10.26456/vtpmk120

Использование Общероссийского математического портала Math-Net.Ru подразумевает, что вы прочитали и согласны с пользовательским соглашением

http: //www.mathnet.ru/rus/agreement

Параметры загрузки:

IP : 54.198 .187 .58

26 апреля 2023 г., 09:30:58 
УДК 519.216

\section{ОБ ОДНОМ ПРИМЕНЕНИИ ДРОБНОГО ДВИЖЕНИЯ ЛЕВИ К МОДЕЛИРОВАНИЮ СЕТЕВОГО ТРАФИКА}

Сидорова О.И.

Тверской государственный университет, г. Тверь

Поступила в редакиию 16.01.2017, после переработки 14.03.2017.

Марковские процессы, хорошо зарекомендовавшие себя при моделировании текстовых и голосовых потоков информации, не способны отразить высокую вариабельность пакетного трафика вкупе с наличием долгой памяти. Эти модели существенно недооценивают реальную нагрузку и характеристики производительности систем. Поэтому построение более адекватных моделей трафика и исследование их свойств остается на сегодняшний день весьма актуальной задачей. В настоящей работе найдена неасимптотическая верхняя граница для длины очереди в системе с неограниченным накопителем и входящим трафиком, характеризующимся фрактальным движением Леви. Расчеты опираются на принципы сетевого анализа с помощью огибающих кривых и не предполагают стационарных режимов функционирования или асимптотик «большого буфера» и «большого числа источников».

Ключевые слова: дробное броуновское движение, $\alpha$-устойчивый субординатор, самоподобные процессы, огибающие процессы, длина очереди.

Вестник ТвГУ. Серия: Прикладная математика. 2017. № 1. С. 17-29.

\section{Введение}

Самоподобный характер и долгая память у трафика в современных телекоммуникационных системах подтверждены многочисленными эмпирическими и аналитическими исследованиями [5,8]. Наличие этих свойств объясняют тяжелыми хвостами у распределений длин сообщений [6]. Наиболее популярными моделями, описывающими самоподобный трафик, являются Дробное Броуновское Движение и Устойчивое Движение Леви $[10,14]$. В таких условиях стандартные методы оценки характеристик обслуживания (QoS), в том числе емкости буферов, числа и пропускной способности каналов, времен ожидания и т.п. требуют пересмотра из-за существенной недооценки предлагаемой нагрузки

$\mathrm{K}$ настоящему моменту времени существует большое количество работ, посвященных анализу характеристик $Q \circ S$ для самоподобных моделей трафика в системах с разной конфигурацией и разными схемами обслуживания. Во многих из них получены оценки для вероятностей переполнения буфера и потери нагрузки, 
когда объем буфера $h \rightarrow \infty$. Однако проверить аккуратность этих результатов затруднительно из-за их асимптотического характера. Поэтому сохраняется интерес и к неасимптотическим оценкам.

\section{1. Необходимые сведения}

Процессы Леви широко используются при описании входящих потоков в различных телекоммуникационных системах. Ниже приводится определение подобных процессов и дается описание их необходимых свойств.

\section{1 Устойчивые распределения и процессы Леви}

Определение 1. Случайный процесс $Y=(Y(t), t \geqslant 0)$ называется процессом Леви, если выполнены условия:

1. $Y(0)=0$ почти наверное;

2. Y имеет независимые и однородные (по времени) приращения;

3. Y является стохастически непрерывным;

4. траектории $Y$ непрерывны справа и имеют конечные пределы слева при $t>0$.

В силу независимости и однородности приращений, распределение процесса $Y$ полностью и единственным образом определяется распределением с.в. $Y(1)$, которое обладает свойством безграничной делимости.

Один из наиболее известных процессов Леви - броуновское движение (винеровский процесс).

Определение 2. Процесс Леви $B=(B(t), t \geqslant 0)$ со значениями в $R^{1}$ называется процессом броуновского движения (BM), если для любых $t \geqslant 0, h>0$ приращение $B(t+h)-B(t)$ имеет гауссовское распределение с нулевым средним и дисперсией $\sigma^{2} \cdot h$.

Если $\sigma^{2}=1$, то имеет место стандартное броуновское движение. Нетрудно показать, что

$$
K(t, s)=\operatorname{Cov}(Y(t), Y(s))=\sigma^{2} \min (t, s) .
$$

Приращения броуновского движения имеют нормальное распределение. В силу центральной предельной теоремы такие распределения получаются асимптотически для нормированных сумм независимых и одинаково распределенных случайных величин с конечной дисперсией. В случае бесконечных дисперсий приходим к понятию устойчивого распределения.

Определение 3. Распределение вероятностей $F$ называется устойчивы.м, если для любых н.о.р.с.в. $X_{1}, X_{2}, X_{3}$, имеющих распределение $F$ и любых положительных $a_{1}$ и $a_{2}$ существуют $a_{3}>0 u c \in R^{1}$ такие, что

$$
a_{1} X_{1}+a_{2} X_{2} \stackrel{d}{=} a_{3} X_{3}+c
$$


где $\stackrel{d}{=}$ означает равенство по распределению.

Если с $=0$ для всех $a_{1}, a_{2}>0$, то распределение называется строго устойчивъм.

Характеристическая функция для устойчивой случайной величины $X$ имеет вид:

$$
E e^{i t X}= \begin{cases}\exp \left\{-\sigma^{\alpha}|t|^{\alpha}\left(1-i \beta \operatorname{sign}(t) \operatorname{tg}\left(\frac{\pi \alpha}{2}\right)\right)+i \mu t\right\}, & \text { если } \quad \alpha \neq 1, \\ \exp \left\{-\sigma|t|\left(1+i \beta \frac{2}{\pi} \operatorname{sign}(t) \ln |t|\right)+i \mu t\right\}, & \text { если } \quad \alpha=1,\end{cases}
$$

где $0<\alpha \leqslant 2-$ показатель устойчивости; $\beta \in[-1,1]-$ параметр асимметрии; $\sigma \geqslant 0$ - параметр масштаба; $\mu \in R^{1}-$ параметр сдвига.

При $\beta=0$ имеем симметричное относительно $\mu$ устойчивое распределение, характеристическая функция которого при $\mu=0$ имеет вид

$$
E e^{i t X}=\exp \left\{-\sigma^{\alpha}|t|^{\alpha}\right\}
$$

Характеристический параметр $\alpha$ отвечает за скорость убывания хвоста распределения. Случай $\alpha=2$ соответствует нормальному распределению - единственному из устойчивых законов с конечными математическим ожиданием и дисперсией. При $0<\alpha<2$ распределение с.в. $X$ имеет тяжёлый хвост, поскольку при $x \rightarrow \infty$ вероятность попадания в хвост убывает степенным образом, т.е.

$$
P(X>x) \sim c_{\alpha} \cdot \sigma^{\alpha} \cdot \frac{1+\beta}{2} \cdot x^{-\alpha}, \quad c_{\alpha}=\frac{\sin (\pi \alpha / 2)}{\pi} \Gamma(\alpha) .
$$

Если $0<\alpha<1, \mu=0$ и $\beta=1$, то случайная величина $X$ положительна с вероятностью 1 . В дальнейшем будем говорить, что случайная величина $X$ имеет стандартное $\alpha$-устойчивое распределение, если $\mu=0$ и $\sigma=1$.

Полезно отметить следующее важное свойство: если с.в. $X$ имеет $\alpha$-устойчивое распределение с параметром $0<\alpha<2$, то для любого $0<\gamma<\alpha$,

$$
E\left|X_{\alpha}\right|^{\gamma}<\infty \quad \text { и } E\left|X_{\alpha}\right|^{\alpha}=\infty .
$$

Поэтому при $0<\alpha<2$ дисперсия с.в. $X$ и моменты порядка $\gamma>2$ бесконечны и, кроме того, при $0<\alpha<1$ математическое ожидание с.в. $X$ также бесконечно.

Определение 4. Случайный процесс $L_{\alpha}=\left(L_{\alpha}(t), t \geqslant 0\right)$ со значениями в $R^{1}$ называется $\alpha$-устойчивым движением Леви, если это процесс Леви, для которого $L_{\alpha}(1)$ имеет заданное устойчивое распределение.

Если у распределения $L_{\alpha}(1), 0<\alpha<1, \beta=1, \mu=0$, то траектории процесса $L_{\alpha}$ являются положительными и неубывающими. Такой процесс называется $\alpha$ устойчивым субординатором.

Если $\alpha=2, \mu=0$, то мы вновь возвращаемся к процессу броуновского движения $B$. 


\section{2 Самоподобные процессы}

Определение 5. Случайный процесс $X=(X(t), t \geqslant 0)$ называется самоподобным с показателем Хёрста $H \geqslant 0$, если выполнено следующее условие

$$
X(c t) \stackrel{d}{=} c^{H} X(t), \quad \forall t \geqslant 0, c>0
$$

где $\stackrel{d}{=}$ означает равенство конечномерных распределений.

Двумя наиболее популярными примерами самоподобных процессов являются дробное броуновское движение $(F B M)$ и $\alpha$-устойчивое движение Леви.

Определение 6. Дробным броуновским движением с параметром Херста Н называется гауссовский процесс $\left(B_{H}(t), t \geqslant 0\right)$ с нулевым средним и ковариационной фнкиией

$$
K_{H}(t, s)=\frac{1}{2}\left[|t|^{2 H}+|s|^{2 H}-|t-s|^{2 H}\right] .
$$

При $H=1 / 2$ мы возвращаемся к обычному броуновскому движению.

Определение $\alpha$-устойчивого движения Леви было дано выше. Этот процесс является самоподобным с параметром $H=1 / \alpha$, поскольку при $x \rightarrow \infty$

$$
P\left(L_{\alpha}(t)>x\right)=P\left(t^{1 / \alpha} L_{\alpha}(1)>x\right) \sim c_{\alpha} \cdot t \cdot x^{-\alpha} .
$$

Дополнительную информацию об устойчивых и самоподобных процессах можно найти в книгах [7] и [13].

\section{3 Фрактальное движение Леви}

Пусть $\left(B_{H}(t), t \in R^{1}\right)$ есть дробное броуновское движение с параметром Херста $H,\left(L_{\alpha}^{1}(t), t \geqslant 0\right),\left(L_{\alpha}^{2}(t), t \geqslant 0\right)$ - стандартные $\alpha$-устойчивые субординаторы, $0<\alpha<1$; процессы $B_{H}, L_{\alpha}^{1}$ и $L_{\alpha}^{2}$ независимы. Следуя [11], дадим следующее определение.

Определение 7. Фрактальным дробным движением Леви называется случайный процесс $X=\left(X(t), t \in R^{1}\right)$ со значениями в $R^{1}$ такой, что

$$
X(t):=\left\{\begin{array}{cc}
B_{H}\left(L_{\alpha}^{1}(t)\right), & t \geqslant 0, \\
B_{H}\left(L_{\alpha}^{2}(-t)\right), & t<0 .
\end{array}\right.
$$

В [11] было доказано, что такой процесс является самоподобным с параметром Херста $H_{1}=H / \alpha$, имеет стационарные приращения и

$$
X(t) \stackrel{d}{=}\left(L_{\alpha}^{1}(t)\right)^{H} Y, \quad \forall t>0,
$$

где $L_{\alpha}^{1}(t)$ и $Y$ есть независимые случайные величины и $Y$ имеет стандартное нормальное распределение.

В работе [1] были описаны свойства многомерного аналога фрактального движения Леви.

Пусть $\left(B_{H}(t), t \in R^{1}\right)$ есть многомерное дробное броуновское движение с параметром Херста $H$ и матрицей ковариаций $\Sigma ;\left(L_{\alpha}^{1}(t), t \geqslant 0\right),\left(L_{\alpha}^{2}(t), t \geqslant 0\right)-$ стандартные $\alpha$-устойчивые субординаторы, $0<\alpha<1$; процессы $B_{H}, L_{\alpha}^{1}$ и $L_{\alpha}^{2}$ независимы. 
Определение 8. Многомерным дробным движением Леви называется случайный процесс $X=\left(X(t), t \in R^{1}\right)$ со значениями в $R^{d}$ такой, что

$$
X(t):=\left\{\begin{aligned}
B_{H}\left(L_{\alpha}^{1}(t)\right), & t \geqslant 0 \\
B_{H}\left(L_{\alpha}^{2}(-t)\right), & t<0 .
\end{aligned}\right.
$$

В [1] было доказано, что такой процесс является самоподобным с параметром Херста $H_{1}=H / \alpha$, имеет стационарные приращения и

$$
X(t) \stackrel{d}{=}\left(\left(L_{\alpha}^{1}(t)\right)^{H_{1}} \cdot Y_{1}, \ldots,\left(L_{\alpha}^{1}(t)\right)^{H_{d}} \cdot Y_{d}\right), \quad \forall t>0
$$

где случайный вектор $Y=\left(Y_{1}, \ldots, Y_{d}\right)$ имеет многомерное нормальное распределение со средним ноль и матрицей ковариаций $\Sigma$, причем $L_{\alpha}^{1}(t)$ и $Y$ независимы.

Параметр Херста $H_{j}$ может быть любым положительным числом. С практической точки зрения наиболее интересен случай $1 / 2<H_{j}<1, j=\overline{1, d}$. В этом случае у процесса $X(t)$ есть конечное математическое ожидание и долгая память, но его приращения имеют распределения, отличные от устойчивых.

Тем не менее можно показать, что хвосты распределений координат вектора $X(1)$ ведут себя в точности так же, как хвосты устойчивых распределений. Поскольку $Y \sim \mathrm{N}(0,1)$, а $Z_{\alpha}=L_{\alpha}^{1}(1)$ имеет стандартное $\alpha$-устойчивое распределение, то по теореме Бреймана [2] для больших $x>0$ и любого $k=\overline{1, d}$ имеем

$$
\begin{gathered}
P\left(\left(Z_{\alpha}\right)^{H_{j}} Y_{j}>x\right)=\frac{1}{2} P\left(\left(Z_{\alpha}\right)^{H_{j}}\left|Y_{j}\right|>x\right) \sim \frac{1}{2} E\left(\left|Y_{j}\right|^{\alpha / H_{j}}\right) \cdot P\left(Z_{\alpha}>x^{1 / H_{j}}\right) \sim \\
\sim \frac{1}{2} c_{\alpha} E\left(\left|Y_{j}\right|^{\alpha / H_{j}}\right) \cdot x^{-\alpha / H_{j}}=c_{j}\left(\alpha, \widetilde{H}_{j}\right) \cdot x^{-1 / \widetilde{H}_{j}}
\end{gathered}
$$

где $\widetilde{H}_{j}=H_{j} / \alpha$ и $c_{j}\left(\alpha, H_{j}\right)=c_{\alpha} E\left(\left|Y_{j}\right|^{\alpha / H_{j}}\right) / 2$.

\section{2. Огибающие процессы}

Полезным инструментом при оценке $Q o S$ являются специальные кривые, которые ограничивают поступающую в систему нагрузку (envelope processes) и определяют гарантии обслуживания (service curves) ( [3]). С их помощью получают неасимптотические границы для длин очередей и задержек в обслуживании. Задавая допустимую вероятность выхода за соответствующие границы, можно найти оптимальные для данной ситуации параметры конфигурации системы. Этот подход более эффективен с точки зрения перераспределения ограниченных сетевых ресурсов, нежели методы, опирающиеся на асимптотические результаты.

Пусть $\{A(t), t \geqslant 0\}-$ это случайный процесс, характеризующий кумулятивную нагрузку, поступившую в систему к моменту $t$. Если трафик обслуживается с постоянной скоростью $r$, а буфер имеет неограниченный объем, то длина очереди к моменту $t$ определяется как

$$
Q(t)=\sup _{s \leqslant t}\{A(s, t)-r(t-s)\}=\sup _{s \leqslant t}\{A(t)-A(s)-r(t-s)\}, \quad t \geqslant 0 .
$$


Определение 9. Огибающая кривая (statistical envelope, se) для процесса $A(t)$ есть неслучайная функиия $\mathcal{G}(t)$, такая, что для любых $s, t \geqslant 0 u \lambda>0$

$$
P(A(s, t)>\mathcal{G}(t-s, \lambda)) \leqslant \varepsilon(\lambda)
$$

где $\varepsilon(\lambda)$ - невозрастающая функция от $\lambda$, удовлетворяющая условию

$$
\varepsilon(\lambda) \rightarrow 0, \quad \lambda \rightarrow \infty .
$$

Таким образом, se-кривая задает верхнюю границу для работы, поступающей в систему на интервале $[s, t]$, выйти за которую можно с маленькой вероятностью (violation probability) $\varepsilon(\lambda)$.

Для трафика, обладающего «короткой памятью», вероятность отклонения величины нагрузки от средней скорости прибытия $m$ убывает экспоненциальным образом (Exponentially Bounded Burstiness, EBB) [15], т.е.

$$
P(A(s, t)>m(t-s)+\lambda) \leqslant K e^{-\theta \lambda},
$$

где $K, m, \theta, \lambda>0$ - некоторые константы.

В [12] было предложено обобщение $E B B$-модели, на случай распределений, имеющих конечные моменты всех порядков (Stochastically Bounded Burstiness, $S B B)$

$$
P(A(s, t)>m(t-s)+\lambda) \leqslant \varepsilon(\lambda),
$$

где функция $\varepsilon(\lambda)$ удовлетворяет свойствам:

1. $\varepsilon(\lambda) \geqslant 0$ для всех $\lambda>0$;

2. если $\lambda_{1} \leqslant \lambda_{2}$, то $\varepsilon\left(\lambda_{1}\right) \geqslant \varepsilon\left(\lambda_{2}\right)$;

3. $\varepsilon_{1}(x)=\int_{x}^{\infty} \varepsilon(u) d u \in \mathcal{F}$ для всех $\varepsilon \in \mathcal{F}$, где $\mathcal{F}-$ класс всех неотрицательных, невозрастающих функций на $[0, \infty)$.

$\mathrm{K}$ этому классу помимо $E B B$-моделей относятся самоподобные процессы, приращения которых имеют легкие хвосты. В частности, для фрактального броуновского движения справедлива оценка

$$
P(A(s, t)>m(t-s)+\lambda) \leqslant K e^{-\theta \lambda^{\alpha}},
$$

где $K>0, \theta>0$ и $0<\alpha<1-$ некоторые константы.

Для описания самоподобного трафика, приращения которого имеют тяжелые хвосты, $S B B$-модель не подходит, поскольку функция $\varepsilon(x)=K x^{-\alpha} \notin \mathcal{F}$. Следуя $[9]$, для произвольного стационарного самоподобного процесса положим

$$
P(A(s, t)>\mathcal{G}(t-s, \lambda))=P\left(A(s, t)>m(t-s)+\lambda(t-s)^{H}\right) \leqslant K \lambda^{-\alpha},
$$

где $K>0, m>0$ - константы, $H \in(0,1)$ - параметр Херста, $\alpha \in(1,2)$ - скорость убывания хвоста распределения. В литературе модель (4) относят к классу $g S B B$ (generalized $S B B$ ). 
Определение 10. Огибающая кривая (statistical sample-path envelope, sspe) для $\{A(s, t)\}_{s \leqslant t}$ есть неслучайнал функиия $\overline{\mathcal{G}}(t)$, такал, что для любых $s, t \geqslant 0 u$ $\lambda>0$

$$
P\left(\sup _{s \leqslant t}\{A(s, t)-\overline{\mathcal{G}}(t-s, \lambda)\}>0\right) \leqslant \bar{\varepsilon}(\lambda)
$$

где $\bar{\varepsilon}(\lambda)$ - невозрастающая функиия от $\lambda$, удовлетворяющая условию

$$
\bar{\varepsilon}(\lambda) \rightarrow 0, \quad \lambda \rightarrow \infty .
$$

Построение соответствующих границ для $E B B$ и $S B B$-моделей описано в [4] и [16]. Для моделей трафика с тяжелыми хвостами в [9] доказано, что

$$
\begin{gathered}
\overline{\mathcal{G}}(t, \lambda)=r t+\lambda, \quad \varepsilon(\lambda)=\widetilde{K} \lambda^{-\alpha(1-H)}, \\
\widetilde{K}=\frac{K}{\alpha H(1-H)} \cdot \inf _{1<\gamma<\frac{r}{m}}\left\{\left(\frac{r}{\gamma}-m\right)^{-\alpha H} \frac{\gamma^{\alpha H(1-H)}}{\log \gamma}\right\},
\end{gathered}
$$

где $r>m-$ скорость обслуживания трафика.

\section{3. Основной результат}

\section{1 Одномернъй случай}

Пусть агрегированная нагрузка $A(t)$, поступившая в систему на интервале $[0, t], t>0$, описывается случайным процессом

$$
A(t):=m t+(\sigma m)^{\widetilde{H}} X(t),
$$

где $m>0$ - средняя скорость прибытия, $\sigma-$ масштабный параметр, $\widetilde{H}=H / \alpha, X(t)$ - фрактальное движение Леви, описанное выше.

Поступивший трафик обслуживается с постоянной скоростью $r>m$, а буферная память неограничена. В этих условиях длина очереди

$$
Q(t)=\sup _{s \leqslant t}\{A(s, t)-r(t-s)\}=\sup _{s \leqslant t}\{A(t)-A(s)-r(t-s)\}, \quad t \geqslant 0
$$

является стационарным процессом.

Пусть нагрузка, поступающая в систему, ограничена. Тогда в силу (1) имеем

$$
P\left(A(t)>m t+\lambda t^{\widetilde{H}}\right)=P\left(\left(Z_{\alpha}\right)^{H} Y>\frac{\lambda t^{\widetilde{H}}}{(\sigma m t)^{\widetilde{H}}}\right) \leqslant K \cdot \lambda^{-\alpha / H}
$$

где $K=K(\alpha, H, \sigma, m) \approx c(\alpha, H) \sigma m$. Данная граница справедлива для $\lambda \rightarrow \infty$. $\mathrm{C}$ практической точки зрения это и есть наиболее интересная область для исследования.

Используя подход, предложенный в [9], дискретизируем временную шкалу, положив $x_{k}=\tau \gamma^{k}, \tau>0, \gamma>1$. Для $t-x_{k} \leqslant s<t-x_{k-1}$ имеем

$$
A(s, t)-r(t-s) \leqslant A\left(t-x_{k}, t\right)-r x_{k-1}
$$


и, следовательно,

$$
Q(t) \leqslant \sup _{k}\left\{A\left(t-x_{k}, t\right)-r x_{k-1}\right\}
$$

Тогда

$$
\begin{gathered}
P(Q(t)>\lambda) \leqslant P\left(\bigcup_{k=-\infty}^{\infty}\left\{A\left(t-x_{k}, t\right)>\lambda+r x_{k-1}\right\}\right) \leqslant \\
\sum_{k=-\infty}^{\infty} P\left(A\left(t-x_{k}, t\right)>\lambda+r x_{k-1}\right)=\sum_{k=-\infty}^{\infty} P\left(\left(Z_{\alpha}\right)^{H} Y>\frac{\lambda+(r / \gamma-m) x_{k}}{\left(\sigma m x_{k}\right)^{\widetilde{H}}}\right) \leqslant \\
\sum_{k=-\infty}^{\infty} K \cdot\left(\frac{\lambda+(r / \gamma-m) x_{k}}{x_{k}^{\widetilde{H}}}\right)^{-\alpha / H} .
\end{gathered}
$$

Применяя лемму 4 из [9], получаем

$$
\begin{aligned}
& \sum_{k=-\infty}^{\infty} K \cdot\left(\frac{\lambda+\left(\frac{r}{\gamma}-m\right) x_{k}}{x_{k}^{\widetilde{H}}}\right)^{-\frac{\alpha}{H}} \leqslant\left.\frac{1}{\widetilde{H}(1-\widetilde{H}) \ln \gamma} \int_{z}^{\infty} K \cdot x^{-\frac{\alpha}{H}-1}\right|_{z=\frac{(r / \gamma-m) \widetilde{H}_{\lambda} 1-\widetilde{H}}{\gamma \tilde{H}(1-\widetilde{H})}} \leqslant \\
& \leqslant \widetilde{K} \cdot \lambda^{-(1-\widetilde{H}) / \widetilde{H}}{ }^{z}
\end{aligned}
$$

Минимизируя полученное выражение по $\gamma \in(1, r / m)$, находим оценку для константы

$$
\tilde{K}=\frac{K}{1-\widetilde{H}} \inf _{1<\gamma<\frac{r}{m}}\left\{\frac{\gamma^{2-\widetilde{H}}}{(r-\gamma m) \ln \gamma}\right\} .
$$

Теорема 1. Для длины очереди в системе с неораниченным накопителем, постоянной скоростью обслуживания и агрегированной нагрузкой, удовлетворяющей условиям (7) и (8), справедливо соотношение

$$
\begin{aligned}
& P(Q(t)>\lambda) \leqslant \widetilde{K} \cdot \lambda^{-(1-\widetilde{H}) / \widetilde{H}}, \quad \lambda \rightarrow \infty, \\
& \widetilde{K}=\frac{K}{1-\widetilde{H}} \inf _{1<\gamma<\frac{r}{m}}\left\{\frac{\gamma^{2-\widetilde{H}}}{(r-\gamma m) \ln \gamma}\right\} \text {. }
\end{aligned}
$$

\section{2 Многомерный случай}

Рассмотрим систему массового обслуживания, в которую поступает нагрузка по нескольким каналам. Величину нагрузки, поступившей по $j$-му каналу, определим по правилу:

$$
A_{j}(t):=m_{j} t+\left(\sigma_{j} m_{j}\right)^{1 / \widetilde{H}_{j}} X_{j}(t), j=\overline{1, d}
$$

Векторный случайный процесс $X(t)=\left(X_{1}(t), \ldots, X_{d}(t)\right)$ есть дробное движение Леви с параметром Херста $\widetilde{H}:=H / \alpha=\left(H_{1} / \alpha, \ldots, H_{d} / \alpha\right)$ и матрицей ковариаций $\Sigma=\left(\sigma_{p q}\right)$, определенный выше. Мы полагаем, что скорость обслуживания нагрузки в $j$-м канале постоянна и равна $r_{j}>m_{j}, j=\overline{1, d}$, а накопители имеют 
неограниченный объем. Тогда величина загрузки $j$-го канала в момент времени $t \in R^{1}$ описывается стационарным процессом вида

$$
Q_{j}(t)=\sup _{s \leqslant t}\left(A_{j}(t)-A_{j}(s)-r_{j} \cdot(t-s)\right), \quad t \geqslant 0 .
$$

Пусть, как и ранее, нагрузка, поступающая в систему по каждому из каналов, ограничена, т.е.

$$
P\left(A_{j}(t)>m_{j} t+\lambda_{j} t^{\widetilde{H}_{j}}\right) \leqslant K_{j} \cdot \lambda_{j}^{-\alpha / H_{j}}, \quad \lambda_{j} \rightarrow \infty,
$$

где $K_{j}=K_{j}\left(\alpha, H_{j}, \sigma_{j}, m_{j}\right) \approx c_{j}\left(\alpha, H_{j}\right) \sigma_{j} m_{j}$.

Положим $\lambda=\lambda_{1}+\ldots+\lambda_{d}$ и $H^{*}=\min _{1 \leqslant j \leqslant d}\left\{\frac{1-\widetilde{H}_{j}}{\widetilde{H}_{j}}\right\}$. Нас будет интересовать вероятность того, что в момент $t$ длина очереди хотя бы в одном из каналов превысит некоторое критическое значение $\lambda_{j}, j=\overline{1, d}$. Имеем

$$
P\left(\bigcup_{j=1}^{d}\left\{Q_{j}(t)>\lambda_{j}\right\}\right) \leqslant \sum_{j=1}^{d} P\left(Q_{j}(t)>\lambda_{j}\right) \leqslant \sum_{j=1}^{d} \widetilde{K}_{j} \cdot \lambda_{j}^{-\left(1-\widetilde{H}_{j}\right) / \widetilde{H}_{j}},
$$

где $\widetilde{K}_{j}$ определены в $(9)$.

Используя тот факт, что

$$
K \lambda^{-\beta} \leqslant K^{\beta^{\prime} / \beta} \lambda^{-\beta^{\prime}}, \quad K \lambda^{-\beta} \leqslant 1, \quad \beta^{\prime}<\beta
$$

и

$$
\min _{\lambda_{1}+\ldots+\lambda_{d}=\lambda} \sum_{j=1}^{d} K_{j} \lambda_{j}^{-\beta}=\left(\sum_{j=1}^{d} K_{j}^{1 /(1+\beta)}\right)^{1+\beta} \lambda^{-\beta},
$$

окончательно получаем оценку

$$
P\left(\bigcup_{j=1}^{d}\left\{Q_{j}(t)>\lambda_{j}\right\}\right) \leqslant\left(\sum_{j=1}^{d} K_{j}^{\frac{H^{*} \widetilde{H}_{j}}{\left(1+H^{*}\right)\left(1-\widetilde{H}_{j}\right)}}\right)^{1+H^{*}} \lambda^{-H^{*}} .
$$

Теорема 2. В рамках описанной выше модели верхняя граница для вероятности того, что хотя бы в одном из каналов длина очереди в момент $t$ превысит некоторое пороговое значение $\lambda_{j}$, имеет следующий вид:

$$
P\left(\bigcup_{j=1}^{d}\left\{Q_{j}(t)>\lambda_{j}\right\}\right) \leqslant\left(\sum_{j=1}^{d} \widetilde{K}_{j}^{\frac{H^{*} \widetilde{H}_{j}}{\left(1+H^{*}\right)\left(1-\widetilde{H}_{j}\right)}}\right)^{1+H^{*}} \lambda^{-H^{*}}, \lambda_{j} \rightarrow \infty, k=\overline{1, d},
$$

əде $\lambda=\lambda_{1}+\ldots+\lambda_{d}$.

\section{Заключение}

На практике обычно интересуются стационарными длинами очередей, т.е. процессами вида

$$
Q_{j}(0)=\sup _{\tau \geqslant 0}\left(A_{j}(\tau)-r_{j} \cdot \tau\right)
$$


На их основе в работах [1] и [11] были получены нижние асимптотические границы для вероятностей переполнения буфера для трафика, порожденного фрактальным движением Леви.

Неасимптотические результаты (9) и (10) также полезны в практических приложениях. В частности, их можно использовать для оценки распределения задержек в обслуживании трафика.

\section{Список литературы}

[1] Хохлов Ю.С. Многомерное дробное движение Леви и его приложения // Информатика и ее применения. 2016. Т. 10, № 2. С. 98-106.

[2] Breiman L. On some limit theorems similar to the arc-sin law // Теория вероятностей и ее применения. 1965. Т. 10, № 2. С. 351-359.

[3] Le Boudec J.Y., Thiran P. A short tutorial on Network Calculus I: fundamental bounds in Communication Networks // Proceedings of 2000 IEEE International Symposium on Circuits and Systems (ISCAS'2000). Geneva, Switzerland, 28-31 May 2000. Pp. 1426-1428.

[4] Ciucu F., Burchard A., Liebeherr J. Scaling properties of statistical end-to-end bounds in the network calculus // IEEE Transactions on Information Theory. 2006. Vol. 52, № 6. Pp. 2300-2312.

[5] Crovella M., Bestavros A. Self-similarity in world wide web traffic: evidence and possible cases // Proceedings of the 1996 ACM SIGMETRICS International Conference on Measurement and Modelling of Computer Systems. 1996. Vol. 4. Pp. 160-169.

[6] Crovella M., Kim G., Park K. On the relationship between file sizes, transport protocols, and self-similar network traffic // Proceedings of the Fourth International Conference on Network Protocols (ICNP'96). Columbus, Ohio, U.S.A, October 29 - November 1, 1996. Pp. 171-180.

[7] Embrechts P., Maejima M. Selfsimilar Process. Prinston University Press, 2002. $111 \mathrm{p}$.

[8] Leland W.E., Taqqu M.S., Willinger W., Willson D.V. On the self-similar nature of Ethernet traffic (Extended version) // IEEE/ACM Transactions on Networking. 1994. Vol. 2. Pp. 1-15.

[9] Liebeherr J., Burchard A., Ciucu F. Delay bounds for networks with heavy-tailed and self-similar traffic // IEEE Transactions on Information Theory. 2012. Vol. 58, № 2. Pp. 1010-1024.

[10] Mikosch Th., Resnick S., Rootzen H., Stegeman A. Is network traffic approximated by stable Levy motion or fractional Brownian motion? // Annals of Applied Probability. 2002. Vol. 12, № 1. Pp. 23-68. 
[11] Nikola C.D., Khokhlov Y.S., Pagano M., Sidorova O.I. Fractional Levy motion with dependent increments and its application to network traffic modeling // Информатика и ее применения. 2012. Т. 6, № 3. С. 59-63.

[12] Starobinski D., Sidi M. Stochastically bounded burstiness for communication networks // IEEE Transactions on Information Theory. 2000. Vol. 46, № 1. Pp. 206-212.

[13] Samorodnitsky G., Taqqu M.S. Stable Non-Gaussian Random Processes. Chapman\&Hall, 1994. 632 p.

[14] Taqqu M.S., Willinger W., Sherman R. Proof of a fundamental result in selfsimilar traffic modeling // Computer Communication Review. 1997. Vol. 27, № 2. Pp. 5-23.

[15] Yaron O., Sidi M. Performance and stability of communication networks via robust exponential bounds // IEEE/ACM Transactions on Networking. 1993. Vol. 1, № 3. Pp. 372-385.

[16] Yin Q., Jiang Y., Jiang S., Kong P.Y. Analysis on generalized stochastically bounded bursty traffic for communication networks // Proceedings of 27th Annual IEEE Conference on Local Computer Networks (LCN 2002). Tampa, Florida, USA, November 6-8, 2002. Pp. 141-149.

\section{Библиографическая ссылка}

Сидорова О.И. Об одном применении дробного движения Леви к моделированию сетевого трафика // Вестник ТвГУ. Серия: Прикладная математика. 2017. № 1. C. 17-29.

\section{Сведения об авторах}

\section{1. Сидорова Оксана Игоревна}

доцент кафедры математической статистики и системного анализа Тверского государственного университета.

Россия, 170100, г. Тверь, ул. Желябова, д. З3, ТвГУ. 


\title{
ON ONE APPLICATION OF FRACTIONAL LEVY MOTION TO NETWORK TRAFFIC MODELING
}

\author{
Sidorova Oksana Igorevna \\ Associate professor at Mathematical Statistics and System Analysis department, \\ Tver State University. \\ Russia, 170100, Tver, 33 Zhelyabova str., TSU.
}

\begin{abstract}
Received 16.01.2017, revised 14.03.2017.
Markovian theory effectively used in modeling of text and voice transmission is not able to reflect the high variability of packet traffic coupled with the presence of long memory. It leads to a substantial underestimation of the network load and a very non-accurate estimation of performance measures. Hence the construction of more adequate models of data flows and analysis of their properties remains a very important task. In this paper we found a non-asymptotic upper bound for queue length in the infinite buffer queue fed by a fractal Levy motion. The analysis follows a network calculus approach where traffic is characterized by envelope functions and do not assume a steady state, large buffer, or many sources regime.
\end{abstract}

Keywords: fractional Brownian motion, $\alpha$-stable subordinator, selfsimilar processes, envelope processes, queue length.

\section{Bibliographic citation}

Sidorova O.I. On one application of fractional Levy motion to network traffic modeling. Vestnik TvGU. Seriya: Prikladnaya Matematika [Herald of Tver State University. Series: Applied Mathematics], 2017, no. 1, pp. 17-29. (in Russian)

\section{References}

[1] Khokhlov Yu.S. The multidimensional fractional Levi motion and its applications. Informatika i ee primeneniya [Informatics and its applications], 2016, vol. 10(2), pp. 98-106. (in Russian)

[2] Breiman L. On some limit theorems similar to the arc-sin law. Teoriya veroyatnostei $i$ ee primeneniya [Theory of Probability and its Applications], 1965, vol. 10(2), pp. 351-359.

[3] Le Boudec J.Y., Thiran P. A short tutorial on Network Calculus I: fundamental bounds in Communication Networks. Proceedings of 2000 IEEE International Symposium on Circuits and Systems (ISCAS'2000). Geneva, Switzerland, 28-31 May 2000. Pp. 1426-1428. 
[4] Ciucu F., Burchard A., Liebeherr J. Scaling properties of statistical end-to-end bounds in the network calculus. IEEE Transactions on Information Theory, 2006, vol. 52(6), pp. 2300-2312.

[5] Crovella M., Bestavros A. Self-similarity in world wide web traffic: evidence and possible cases. Proceedings of the 1996 ACM SIGMETRICS International Conference on Measurement and Modelling of Computer Systems. 1996. Vol. 4. Pp. 160-169.

[6] Crovella M., Kim G., Park K. On the relationship between file sizes, transport protocols, and self-similar network traffic. Proceedings of the Fourth International Conference on Network Protocols (ICNP'96). Columbus, Ohio, U.S.A, October 29 - November 1, 1996. Pp. 171-180.

[7] Embrechts P., Maejima M. Selfsimilar Process. Prinston University Press, 2002. $111 \mathrm{p}$.

[8] Leland W.E., Taqqu M.S., Willinger W., Willson D.V. On the self-similar nature of Ethernet traffic (Extended version). IEEE/ACM Transactions on Networking, 1994, vol. 2, pp. 1-15.

[9] Liebeherr J., Burchard A., Ciucu F. Delay bounds for networks with heavytailed and self-similar traffic. IEEE Transactions on Information Theory, 2012, vol. 58(2), pp. 1010-1024.

[10] Mikosch Th., Resnick S., Rootzen H., Stegeman A. Is network traffic approximated by stable Levy motion or fractional Brownian motion? Annals of Applied Probability, 2002, vol. 12(1), pp. 23-68.

[11] Nikola C.D., Khokhlov Y.S., Pagano M., Sidorova O.I. Fractional Levy motion with dependent increments and its application to network traffic modeling. Informatika $i$ ee primeneniya [Informatics and its applications], 2012, vol. 6(3), pp. 59-63.

[12] Starobinski D., Sidi M. Stochastically bounded burstiness for communication networks. IEEE Transactions on Information Theory, 2000, vol. 46(1), pp. 206212.

[13] Samorodnitsky G., Taqqu M.S. Stable Non-Gaussian Random Processes. Chapman\&Hall, 1994. 632 p.

[14] Taqqu M.S., Willinger W., Sherman R. Proof of a fundamental result in self-similar traffic modeling. Computer Communication Review, 1997, vol. 27(2), pp. 5-23.

[15] Yaron O., Sidi M. Performance and stability of communication networks via robust exponential bounds. IEEE/ACM Transactions on Networking, 1993, vol. 1(3), pp. $372-385$.

[16] Yin Q., Jiang Y., Jiang S., Kong P.Y. Analysis on generalized stochastically bounded bursty traffic for communication networks. Proceedings of 27th Annual IEEE Conference on Local Computer Networks (LCN 2002). Tampa, Florida, USA, November 6-8, 2002. Pp. 141-149. 